\title{
Histone deacetylase 6 acts upstream of DNA damage response activation to support the survival of glioblastoma cells
}

\author{
Wen-Bin Yang ${ }^{1}{ }^{1,2,3}$, An-Chih Wu ${ }^{2}$, Tsung-I Hsu ${ }^{1,3,4,5}$, Jing-Ping Liou ${ }^{6,7}$, Wei-Lun Lo ${ }^{8,9}$, Kwang-Yu Chang ${ }^{10}$, Pin-Yuan Chen ${ }^{11}$, \\ Ushio Kikkawa $^{2}$, Shung-Tai Yang ${ }^{8}$, Tzu-Jen Kao ${ }^{1,3}$, Ruei-Ming Chen ${ }^{2,4}$, Wen-Chang Chang ${ }^{2,3}$, Chiung-Yuan Ko (iD ${ }^{1,3,4}$ and $^{\circ}$ \\ Jian-Ying Chuang (iD) $1,3,4,5,12 \bowtie$
}

(c) The Author(s) 2021

DNA repair promotes the progression and recurrence of glioblastoma (GBM). However, there remain no effective therapies for targeting the DNA damage response and repair (DDR) pathway in the clinical setting. Thus, we aimed to conduct a comprehensive analysis of DDR genes in GBM specimens to understand the molecular mechanisms underlying treatment resistance. Herein, transcriptomic analysis of 177 well-defined DDR genes was performed with normal and GBM specimens $(n=137)$ from The Cancer Genome Atlas and further integrated with the expression profiling of histone deacetylase 6 (HDAC6) inhibition in temozolomide (TMZ)-resistant GBM cells and patient-derived tumor cells. The effects of HDAC6 inhibition on DDR signaling were examined both in vitro and intracranial mouse models. We found that the expression of DDR genes, involved in repair pathways for DNA doublestrand breaks, was upregulated in highly malignant primary and recurrent brain tumors, and their expression was related to abnormal clinical features. However, a potent HDAC6 inhibitor, MPTOB291, attenuated the expression of these genes, including RAD51 and CHEK1, and was more effective in blocking homologous recombination repair in GBM cells. Interestingly, it resulted in lower cytotoxicity in primary glial cells than other HDAC6 inhibitors. MPT0B291 reduced the growth of both TMZ-sensitive and TMZresistant tumor cells and prolonged survival in mouse models of GBM. We verified that HDAC6 regulated DDR genes by affecting Sp1 expression, which abolished MPTOB291-induced DNA damage. Our findings uncover a regulatory network among HDAC6, Sp1, and DDR genes for drug resistance and survival of GBM cells. Furthermore, MPTOB291 may serve as a potential lead compound for GBM therapy.

Cell Death and Disease (2021)12:884; https://doi.org/10.1038/s41419-021-04182-w

\section{INTRODUCTION}

Glioblastoma (GBM), the most common adult malignant brain tumor, is one of the deadliest cancers, as it involves a highly aggressive feature and causes poor prognosis even after simultaneous standard treatment with radiation and temozolomide (TMZ)-based chemotherapy [1, 2]. TMZ is an imidazotetrazine derivative of an alkylating agent and acts as a DNA methylating agent, thereby resulting in DNA lesion (06-methylguanine, N7-methylguanine, and N3-methyladenine) and DNA mismatches to cause cellular senescence and apoptosis [3]. Promoter methylation of the DNA repair gene, 0-6methylguanine-DNA methyltransferase (MGMT), which acts by removing alkyl groups from DNA, has been associated with longer survival in response to TMZ treatment [4]. Induction of MGMT expression is frequently associated with TMZ treatment at recurrence. However, resistance to TMZ also occurs in MGMTnegative GBM cells, indicating the involvement of other factors $[3,5]$.

In clinical practice, patients with isocitrate dehydrogenase $(\mathrm{NADP}(+)) 1(\mathrm{IDH} 1)^{\mathrm{R} 132 \mathrm{H}}$ mutation, a diagnostic marker and prognostic indicator of GBM, gain survival benefit in contrast to those without the mutation in both primary and secondary GBM $[6,7]$. IDH1 is a key enzyme that catalyzes the conversion of isocitrate to a-ketoglutaric acid (aKG) in the tricarboxylic acid cycle. IDH $1^{\mathrm{R} 132 \mathrm{H}}$ mutation acquires neomorphic ability to produce D-2-hydroxyglutarate from aKG, causing cellular alterations resulting in metabolic dysfunction and inhibition of DNA repair [6]. Several studies shed light on the impact of $I D H 1^{\mathrm{R} 132 \mathrm{H}}$ mutation with impaired DNA repair mechanisms, including inhibition of DNA repair enzyme alkB homolog 1, histone $\mathrm{H} 2 \mathrm{~A}$ dioxygenase

\footnotetext{
${ }^{1}$ TMU Research Center of Neuroscience, Taipei Medical University, 11031 Taipei, Taiwan. ${ }^{2}$ Graduate Institute of Medical Sciences, College of Medicine, Taipei Medical University, 11031 Taipei, Taiwan. ${ }^{3}$ The Ph.D. Program for Neural Regenerative Medicine, College of Medical Science and Technology, Taipei Medical University, 11031 Taipei, Taiwan. ${ }^{4}$ TMU Research Center of Cancer Translational Medicine, Taipei Medical University, 11031 Taipei, Taiwan. ${ }^{5}$ Cell Physiology and Molecular Image Research Center, Wan Fang Hospital, Taipei Medical University, 11031 Taipei, Taiwan. ${ }^{6}$ School of Pharmacy, College of Pharmacy, Taipei Medical University, 11031 Taipei, Taiwan. ${ }^{7}$ TMU Research Center of Drug Discovery, Taipei Medical University, 11031 Taipei, Taiwan. ${ }^{8}$ Department of Neurosurgery, Shuang Ho Hospital, Taipei Medical University, 23561 New Taipei City, Taiwan. ${ }^{9}$ Department of Surgery, School of Medicine, College of Medicine, Taipei Medical University, 11031 Taipei, Taiwan. ${ }^{10}$ National Institute of Cancer Research, National Health

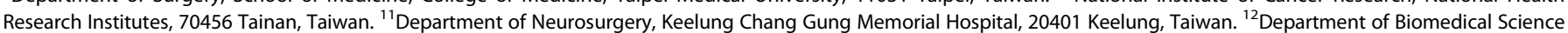
and Environmental Biology, Kaohsiung Medical University, 80708 Kaohsiung, Taiwan. ${ }^{凶}$ email: ko680108@tmu.edu.tw; chuangcy@tmu.edu.tw Edited by Professor Bertrand Joseph
}

Received: 17 June 2021 Revised: 29 August 2021 Accepted: 16 September 2021

Published online: 28 September 2021 
(ALKBH) as well as defects of homologous recombination (HR) DNA repair $[8,9]$. HR is one of the two major DNA double-strand break (DSB) repair pathways, which repairs broken DNA caused by oncogene-induced replication stress, ionizing radiation, or chemotherapeutic drugs [10]. DNA damage response and repair (DDR) signaling is considered to be a barrier for tumor formation and drug resistance $[11,12]$. These findings provide a clue that targeting the general DDR signaling is crucial as IDH1-mutated features may be potential targets in most patients with GBM.

Several abnormally expressed-DDR genes, including exonuclease 1 (EXO1), nei-like DNA glycosylase 3 (NEIL3), and DNA damage-binding protein 2 (DDB2), are associated with GBM progression and susceptibility [13]. Poly-ADP-ribose polymerase (PARP) is an abundant nuclear enzyme involved in base excision repair (BER), and a PARP inhibitor is currently administered in adult patients with unresectable or partially resectable GBM in a clinical trial [14]. The clinical effectiveness of therapies targeting these genes is, however, still rare. Namely, there is a lack of a systemic approach that integrates the genome-wide landscape of expression profiling, big data analytics, and clinical significance, which might be critical issues in translational research for the application of DNA repair blockade.

Histone deacetylase 6 (HDAC6) belongs to the class llb of HDAC family and is regulated by several protein kinases, including protein kinase $C$ alpha [15], extracellular signal-regulated kinase [16], G protein-coupled receptor kinase 2 [17], casein kinase 2 [18], aurora A kinase [19], etc., which lead to HDAC6 activation and its downstream targets in altering multiple cellular processes. HDAC6 is considered to play a unique role owing to its cytoplasmic localization and ability to deacetylate non-histone proteins [20]. HDAC6 affects the dynamics of the cellular structure through deacetylating a-tubulin in the assembled microtubules [21]. Recent studies have highlighted the importance of the nuclear functions of HDAC6 in GBM progression and recurrence. HDAC6, which is overexpressed in clinical GBM tumors [22], TMZ-resistant GBM cells and GBM stem-like tumorspheres [23], affects the expression of cell-cycle-related genes and cancer stemness-related genes through regulating acetylation levels of Sp1 transcription factor $[23,24]$. Furthermore, it has been reported that HDAC6 deacetylates mutS homolog 2 (MSH2), a key DNA mismatch repair (MMR) protein [25]. Inhibition of HDAC6 correlates with increased expression of MSH2 in TMZ-resistant GBM cells [26]; however, whether HDAC6 controls function in DNA repair through MMR remains unknown. Further, there is still a lack of comprehensive analysis to elucidate the mechanism of HDAC6-mediated DNA repair signaling in GBM progression and recurrence. Therefore, in this study, we collected human GBM transcriptome data from The Cancer Genome Atlas (TCGA-GBM dataset) and performed a systemic analysis for characterizing the functional role of HDAC6 in DDR pathways, including not only HR, BER, MMR, but also nucleotide excision repair (NER), nonhomologous end joining (NHEJ), etc. Also, we used in vitro experiments and intracranial mouse models for targeting HDAC6 via pharmacological approaches and genetic knockdown to further examine the role of HDAC6 on the DDR regulation in both TMZ-sensitive and TMZresistant GBM.

\section{RESULTS \\ Inhibition of HDAC6 reverses abnormal clinical features of DDR genes in GBM}

To investigate the DDR gene expression in clinical GBM tumors, we collected RNA-seq data of 137 GBM samples from the TCGA database. By further analysis of 177 well-defined DDR genes using hierarchical clustering, we identified 25 DDR genes (the clustered DDR genes) that were significantly $(p<0.05)$ upregulated in primary and recurrent GBM (Fig. 1A).
Our recent study have indicated that MPTOB291, a potent HDAC6 inhibitor, induces G2/M arrest and senescence in both parental and TMZ-resistant GBM cells [23]. Here, we further analyzed whether the pathway of "DNA replication, recombination, and repair" is underlying MPTOB291-induced growth inhibition of GBM cells. The DDR gene expression profiling following the MPTOB291 treatment of TMZ-resistant U87MG (U87MG-R) or patient-derived P3 (P3-R) cells was performed. The comparison of the expression of DDR genes between MPTOB291-treated cells and TCGA-GBM dataset revealed a negative correlation (Supplementary Fig. S1A, PCC $=-0.272$ ), especially in the clustered DDR genes (Supplementary Fig. S1B, PCC $=-0.412$ ). The reduction of the DDR gene expression was observed following MPTOB291 treatment (Fig. 1B), in which 14 and 5 DDR genes in U87MG-R and P3-R cells, respectively (Fig. 1C) exhibited converse changes of those of the clustered DDR genes shown in Fig. 1A. Enrichment analysis of the overlapping genes using MetaCore software (Fig. 1D) identified that the top three process networks were "DSB repair", "MMR repair", and "BER-NER repair". These results suggest that HDAC6 may be involved in regulating the abnormal expression of the DDR genes affecting DNA repair.

\section{Inhibition of HDAC6 decreases DDR gene expression, induces DNA damage, and inhibits the growth of GBM and TMZ- resistant GBM}

Among the significantly altered genes overlapped in TCGA-GBM dataset and MPTOB291-treated GBM cells, four genes (RAD51, $\mathrm{CHK} 1, \mathrm{FANCl}$, and $\mathrm{FANCD} 2$ ) were the most potential ones involved in the regulation of HDAC6-mediated DNA repair (Fig. 2A). Furthermore, the protein expression of RAD51 recombinase (catalyzes the core reactions of HR) and CHEK1 (a kinase central for the DDR signaling at the $S$ and G2/M cell cycle checkpoints) was examined, and dose-related decreases of both protein levels were observed after MPTOB291 treatment in A172 GBM cells and TMZ-resistant A172 GBM cells (Fig. 2B), as well as in the patientderived TMZ-resistant GBM cells (Fig. 2C). Also, we found a corresponding increase in the levels of phospho-histone H2A.X $(\mathrm{\gamma H} 2 \mathrm{AX}$, a marker for DNA damage) and acetyl-a-tubulin (actubulin, a marker for HDAC6 inhibition) in these cells (Fig. 2B, C). An increase in the frequency of micronuclei cells relative to the control group (Fig. 2D) indicated that MPTOB291 induces unrepaired DSB or mitotic spindle damage. Supporting this finding, we observed that MPTOB291 impaired DSB repair by blocking HR repair (Fig. 2E, F) rather than by NHEJ (Fig. 2G). To avoid the off-target effects of MPTOB291, we also confirmed the HR efficiency after other commercial HDAC6 inhibitor treatment (including tubacin, nextruastat $A$, and tubastatin A). All of the HDAC6 inhibitors could significantly decrease the repair efficiency of HR. However, MPTOB291 and tubacin showed more effective inhibition of $\mathrm{HR}$ at $2 \mu \mathrm{M}$ concentration. (Fig. 3A, B). DDR genes (RAD51, CHEK1, and $\gamma \mathrm{H} 2 \mathrm{AX}$ ) had the same expression patterns in MPTOB291- and tubacin-treated cells (Fig. 3C). Surprisingly, combined treatment with a lower dosage of HDAC6 inhibitor and TMZ was more effective in triggering DDR than individual treatment (Fig. 3C, D). However, treatment with a higher dosage $(10 \mu \mathrm{M})$ of MPTOB291 could fully induce DDR whether co-treated with TMZ or not (Fig. 3D), suggesting that MPTOB291 is highly effective in inducing DNA damage and is an ideal choice for a single-drug treatment. These findings provide a regulatory mechanism of DNA repair by HDAC6 because DNA damaging agents are widely used in oncology to treat many cancers; therefore, targeting HDAC6 could be a therapeutic strategy for GBM.

We further confirmed the effectiveness of MPTOB291 in GBM treatment. MPTOB291 at concentrations of 2-8 $\mu \mathrm{M}$ did not induce significant cell toxicity in primary glial cells (Fig. 4A); however, it inhibited cell proliferation and induced cell death in both GBM, TMZ-resistant GBM, and patient-derived TMZ-resistant 
A

137 TCGA-GBM RNA-seq data

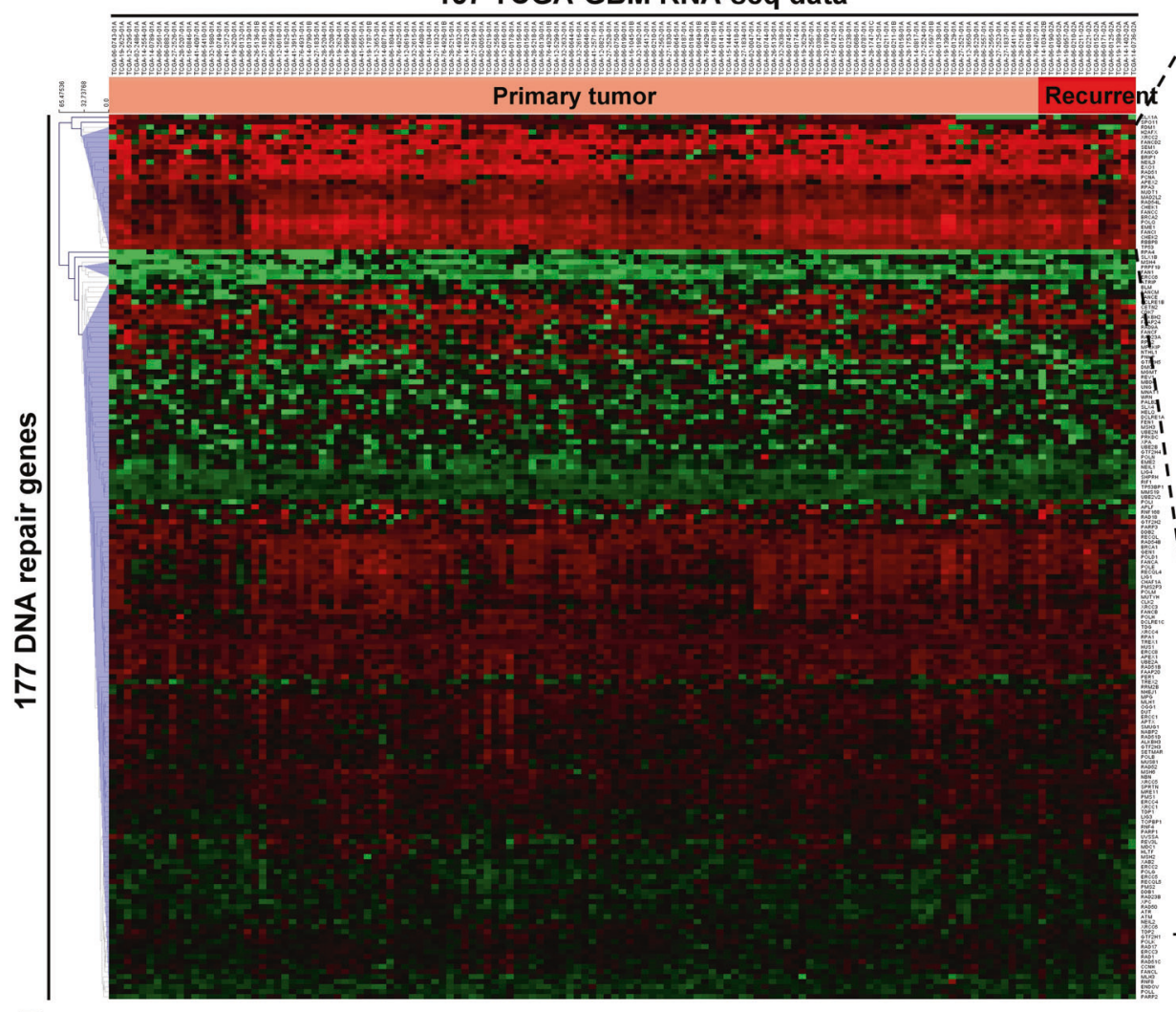

Clustered DDR genes

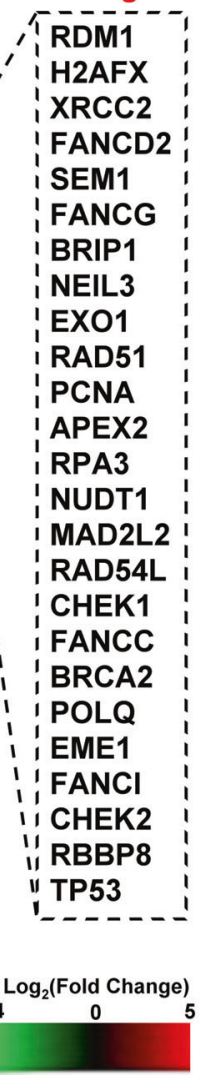

$\mathbf{B}$

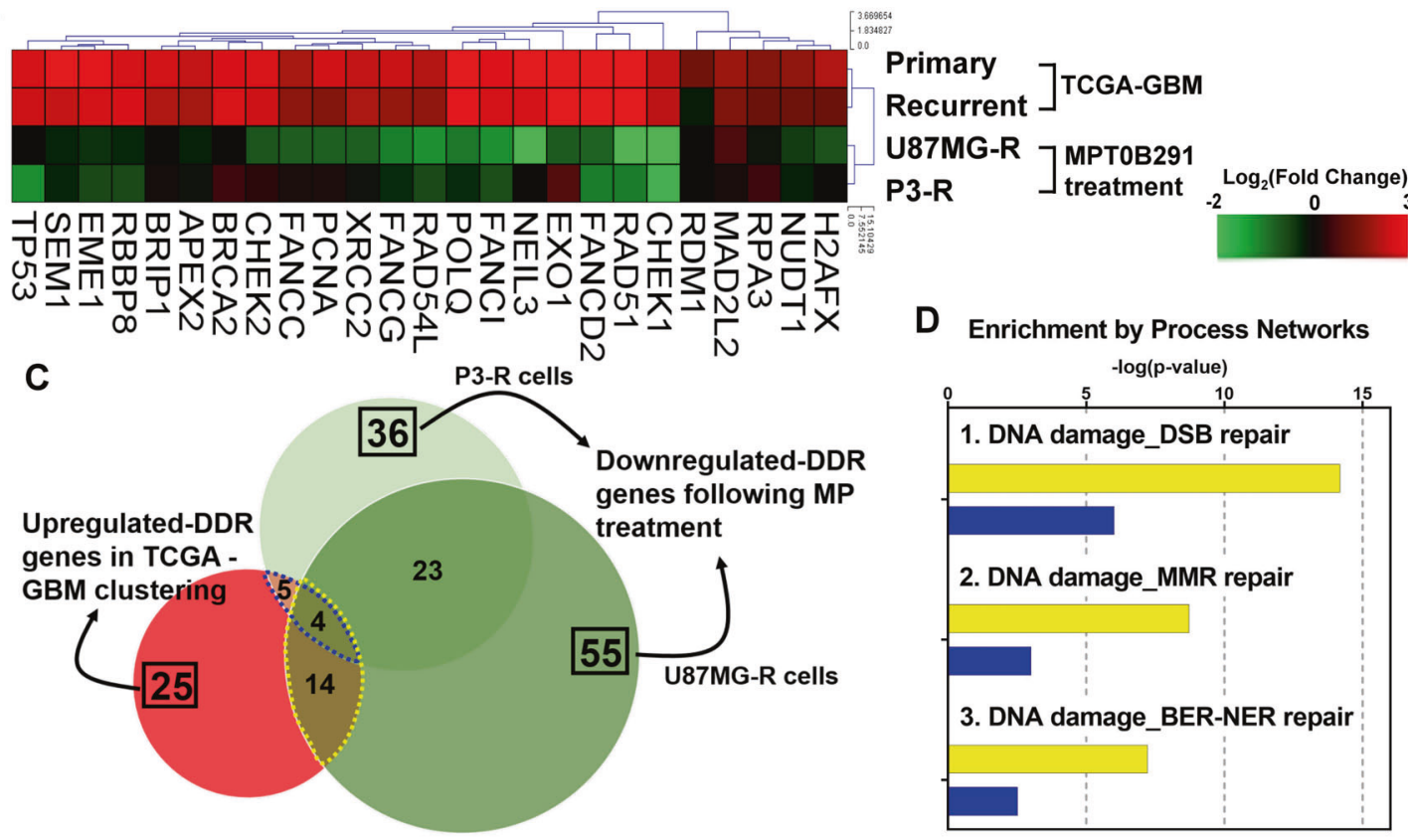

Fig. 1 MPTOB291 reverses abnormal clinical features of DDR genes in GBM. A Heatmap representation of the expression levels of 177 welldefined DDR genes in 137 clinical primary or recurrent GBM samples from the TCGA database. Twenty-five upregulated genes (the clustered DDR genes) compared to solid normal controls were clustered using the Euclidean distance method and shown in the dashed frame on the right panel. B Heatmap representation of fold changes of the clustered DDR genes identified by the above analysis in primary and recurrent tumors versus normal brain tissue and in MPTOB291-treated U87MG-R and P3-R cells versus DMSO-treated parental cells. C Venn diagram showing overlaps between the number of DDR genes that were upregulated (two-fold increase) in TCGA-GBM dataset or downregulated (1.5fold decrease) after MPTOB291 treatment in U87MG-R and P3-R cells. D MetaCore analysis was conducted on the intersected genes between TCGA-GBM dataset and MPTOB291-treated U87MG-R/P3-R cells according to the above analysis to evaluate their functions. The yellow and blue bars show results of 14 and 5 co-regulated DDR genes, respectively. The number of involved genes is shown on the bars. 
A

RAD51

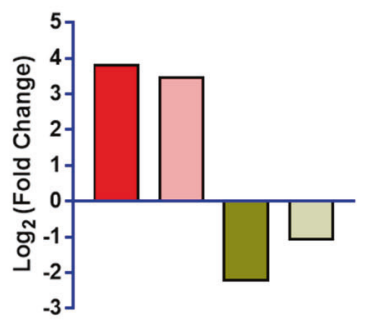

B
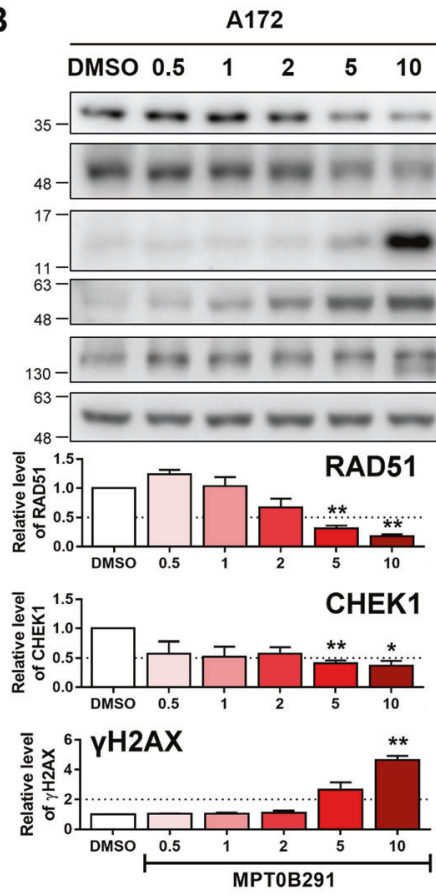

D
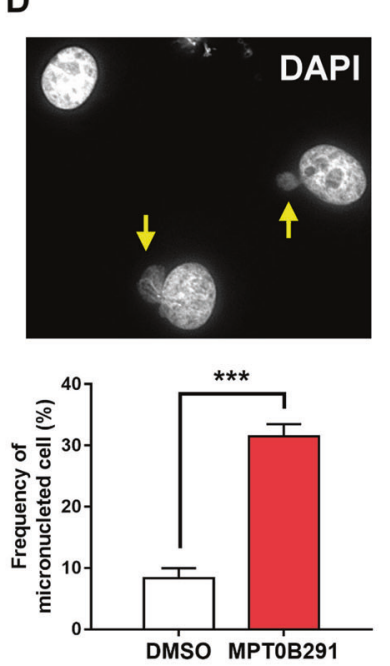

CHEK1

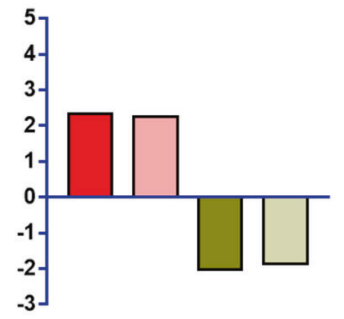

A172-R

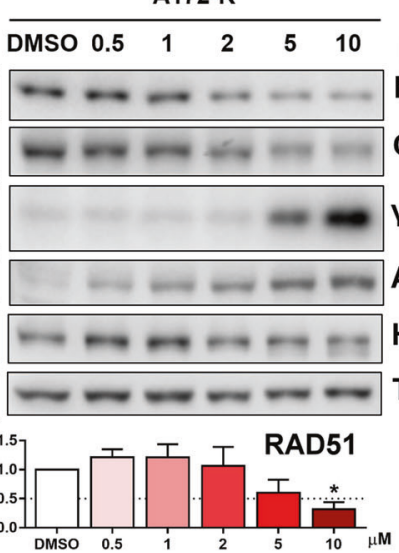

CHEK1
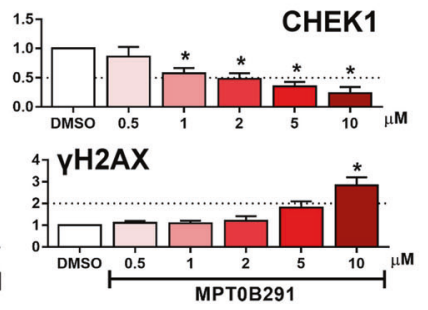

FANCI

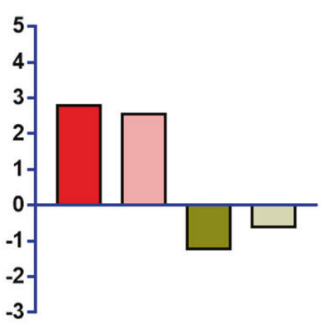

FANCD2

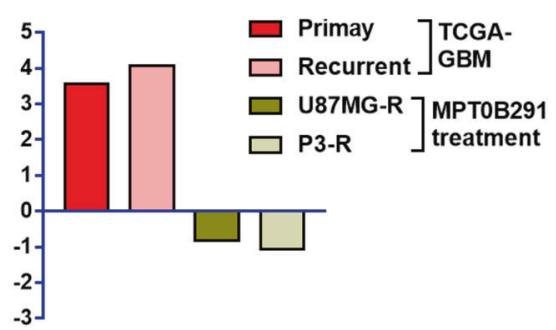

C

$\mu \mathrm{M}$ MP

RAD51

CHEK1

yH2AX

Ac-tubulin

HDAC6

Tubulin
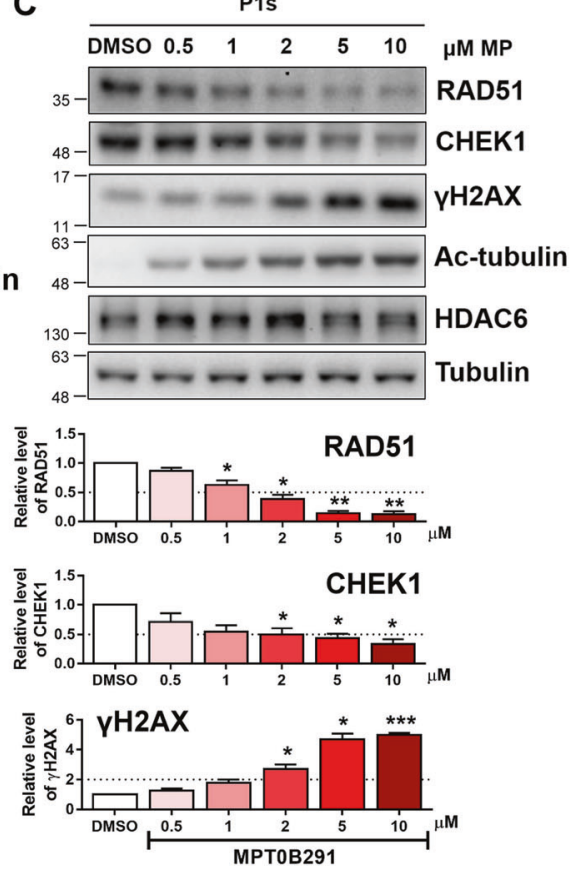

G

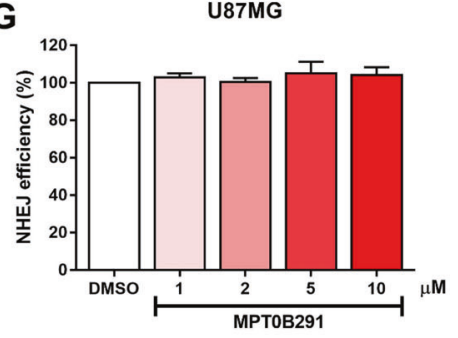

P3

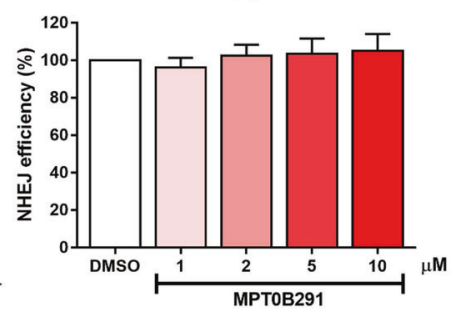

Fig. 2 MPTOB291 decreases DDR gene expression and induces DNA damage. A The fold change in the expression of RAD51, CHEK1, FANCl, and FANCD2 in TCGA-GBM dataset of primary and recurrent tumor samples, and in MPTOB291-treated U87MG-R and P3-R cells. B A172, A172$\mathrm{R}$, and C P1s cells were treated with the indicated doses of MPTOB291 for $24 \mathrm{~h}$, and the protein levels of RAD51, CHEK1, $\gamma \mathrm{H} 2 \mathrm{AX}, \mathrm{Ac}$-tubulin, HDAC6, and tubulin were analyzed by western blotting (upper panels). Quantitative results (normalized to tubulin) of RAD51, CHEK1, and $\gamma \mathrm{H} 2 \mathrm{AX}$ from three independent experiments are shown (lower panels). D U87MG cells were fixed and stained with DAPI following DMSO or MPTOB291 treatment for $24 \mathrm{~h}$. The number of micronuclei (indicated by arrows) was calculated and quantified in more than 600 cells from three independent experiments. E HR assay was performed after the treatment with DMSO or indicated doses of MPTOB291. The percentage of F HR and G NHEJ efficiency was calculated as the ratio of MPTOB291 treatment to DMSO treatment and quantified after three independent experiments. 
A

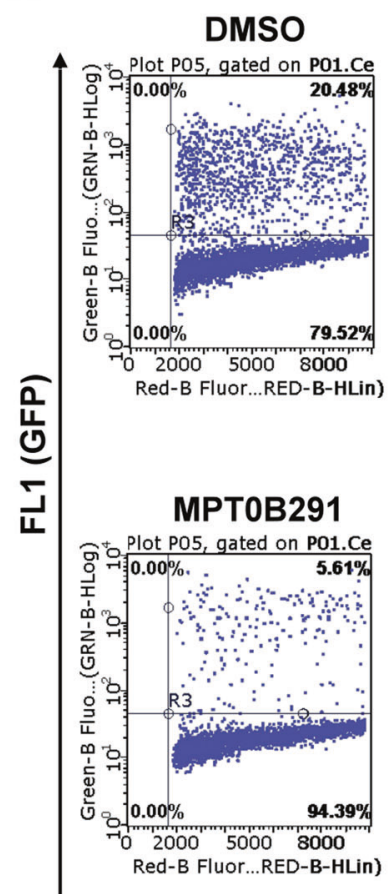

B
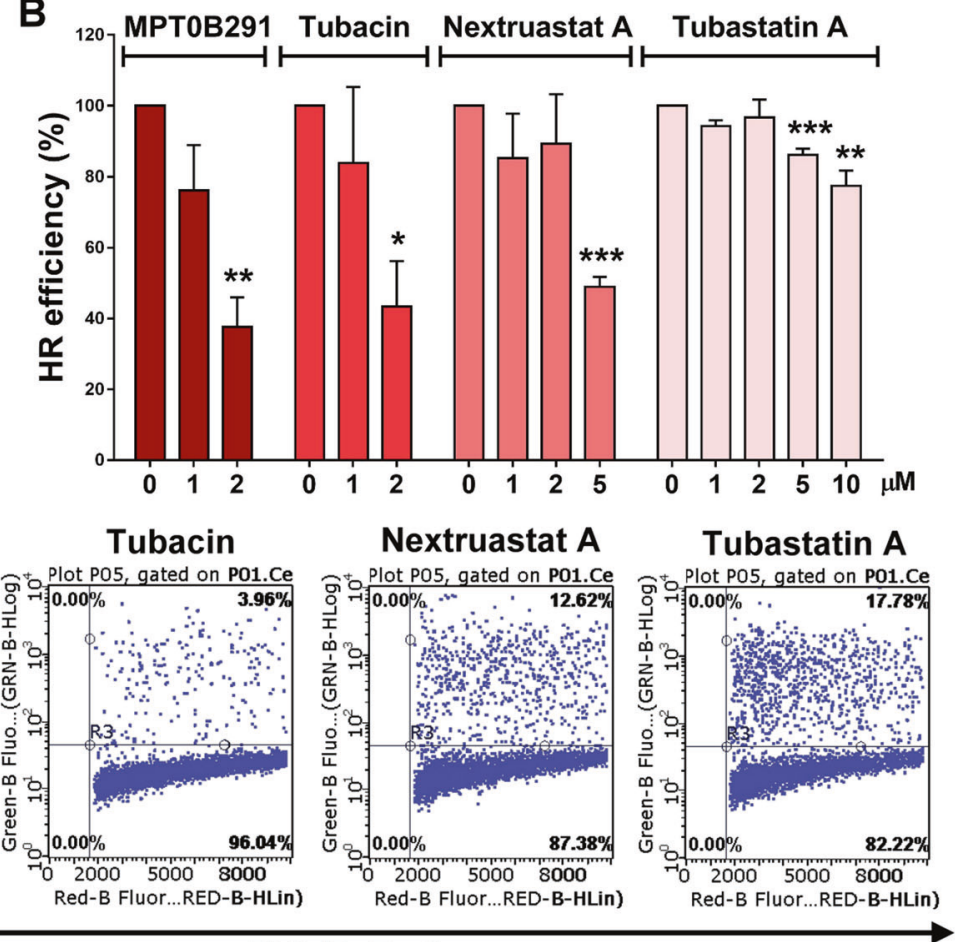

FL3 (DsRed)

C

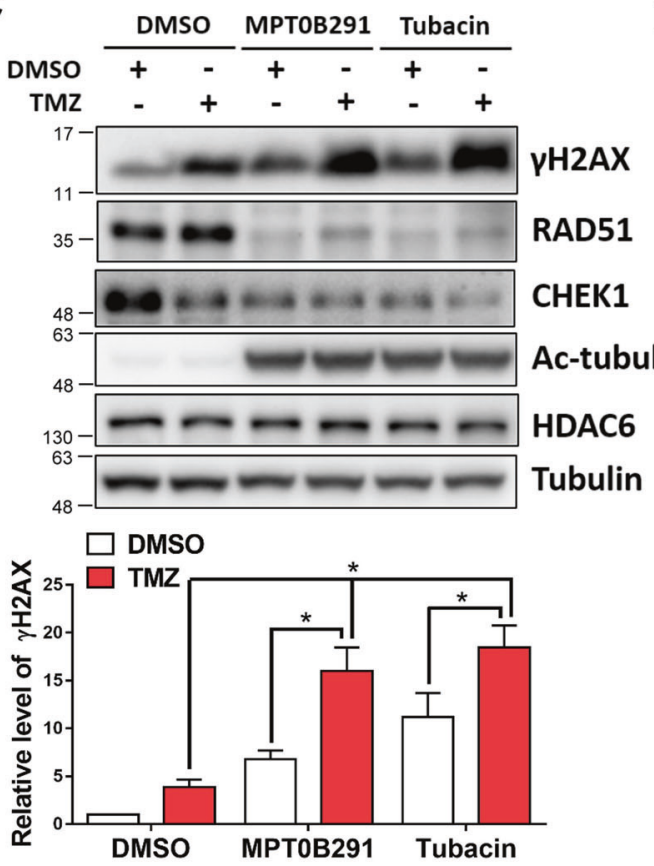

Fig. 3 Inhibition of HDAC6 enhances TMZ-induced DNA damage response. A, B HR assay was performed after the treatment with DMSO or each HDAC6 inhibitor for $48 \mathrm{~h}$ at $2 \mu \mathrm{M}$ in $\mathbf{A}$ and at indicated doses in B. Percentage of HR efficiency was calculated as the ratio of the indicated dosage of HDAC6 inhibitor treatment to DMSO treatment and quantified after three independent experiments. C U87MG cells were treated with $5 \mu \mathrm{M}$ MPTOB291 or tubacin in the presence $(100 \mu \mathrm{M})$ or absence of TMZ for $24 \mathrm{~h}$. D A172 cells were treated with the indicated concentrations of MPTOB291 in the presence $(100 \mu \mathrm{M})$ or absence of TMZ for $24 \mathrm{~h}$. The protein levels of $\gamma \mathrm{H} 2 \mathrm{AX}, \mathrm{RAD} 51, \mathrm{CHEK} 1, \mathrm{HDAC6}, \mathrm{Ac}-$ tubulin, and tubulin were analyzed by western blotting (upper panels). Quantitative results (normalized to tubulin) of $\gamma \mathrm{H} 2 \mathrm{AX}$ from three independent experiments are shown (lower panels).

GBM cells (Fig. 4B). In addition, attenuation of tumor growth was noted in the MPTOB291-treated group (Fig. 4C). MPTOB291 also prolonged mouse survival in orthotopic transplantation models of GBM cells, including TMZ-sensitive and TMZ-resistant cells (Fig. 4D). Activation of the DDR signal could be detected in the MPTOB291-treated group (Fig. 4E). These results indicate that HDAC6 is an attractive therapeutic target in brain tumors. 
A

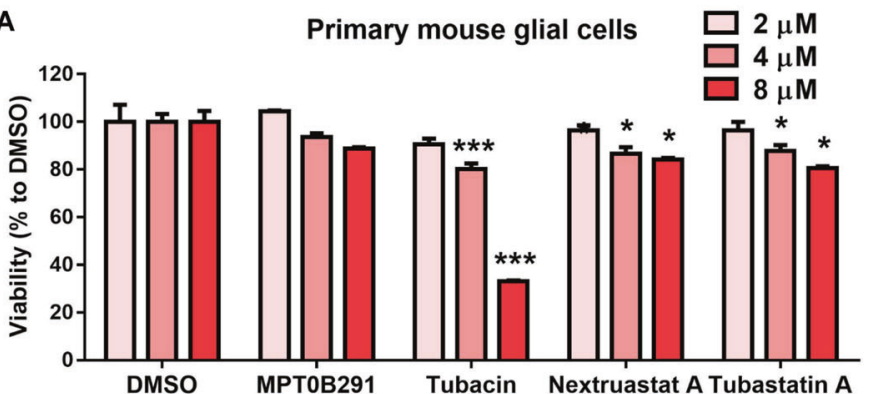

(b)

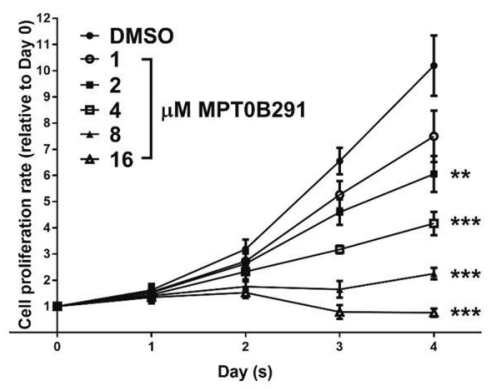

(c)

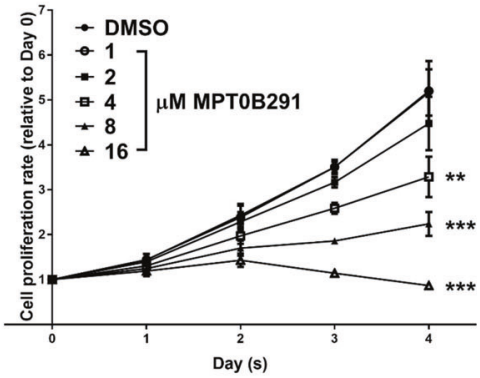

C

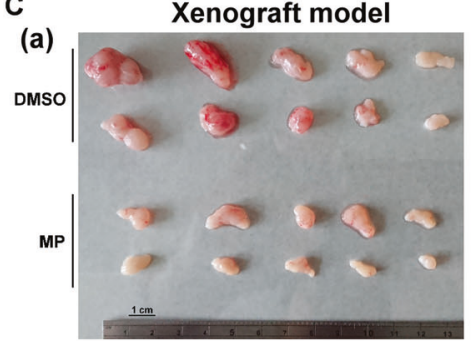

(b)

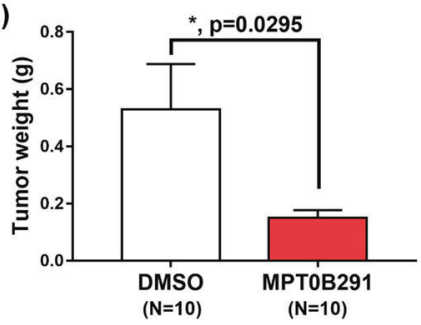

(d)

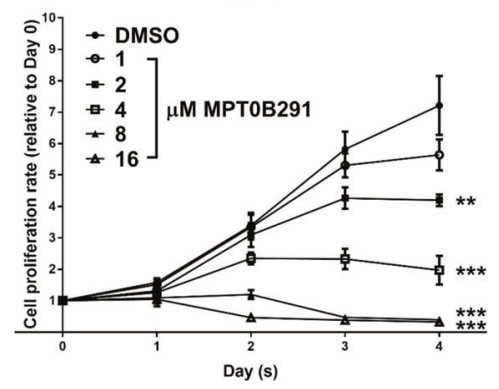

(e)

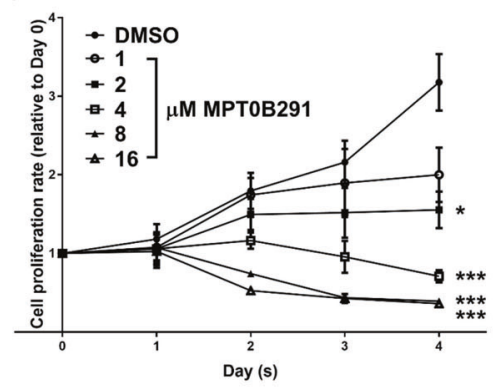

E

(a)

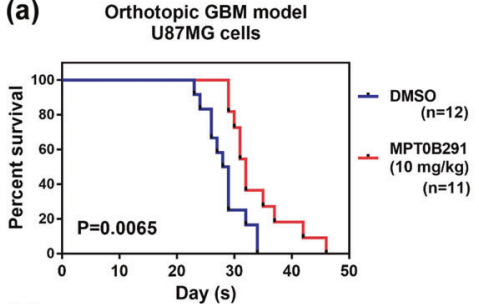

(b)

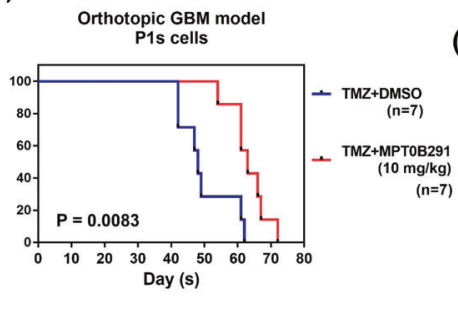

B

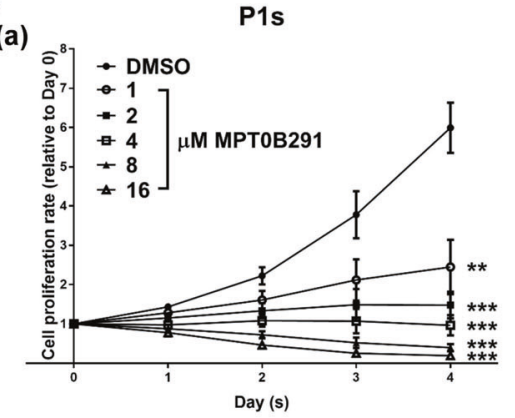

(f)

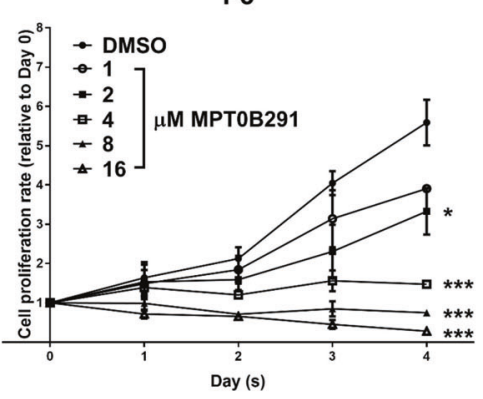

(g)

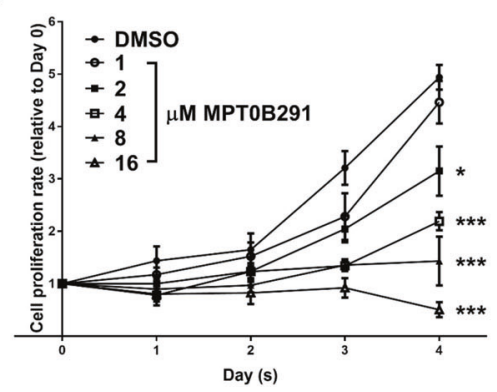

(a)
YH2AX

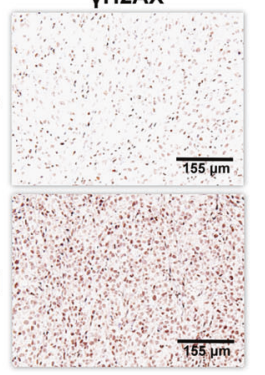

(b)

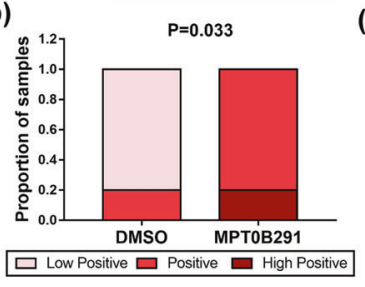

(c) H\&E

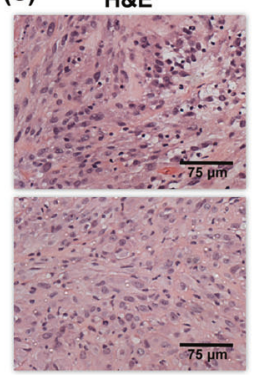

(d) 흘

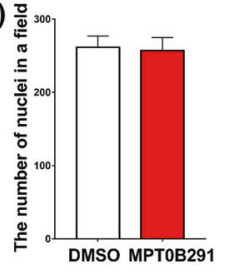

\section{The HDAC6/Sp1 axis mediates the abnormal regulation of DDR genes in GBM}

Although MPTOB291 is a potent and selective HDAC6 inhibitor, with an $\mathrm{IC}_{50}$ value of $5 \mathrm{nM}$, it can also target HDAC1 and HDAC2 at higher treatment concentrations [23]. To elucidate which HDAC is involved in regulating the expression of DDR genes, we used the knockdown approach with siRNA to directly target HDAC1, HDAC2, or HDAC6. Knockdown of HDAC6 significantly decreased the expression of RAD51 but that of HDAC1 and HDAC2 did not (Fig. 5A). This result led to further investigation of possible transcription factors (TFs) involved in the regulation of HDAC6mediated DDR genes. For this purpose, we integrated promoter analysis of dysregulated DDR genes, and expression profiles of TFs in the TCGA-GBM dataset to identify potential TFs. The transcription factor $\mathrm{Sp} 1$ was the most potential TF involved in regulating the abnormal expression of DDR genes, which commonly existed 
Fig. 4 MPTOB291 inhibits the growth of GBM and TMZ-resistant GBM. A Primary mouse glial cells were treated with DMSO or indicated concentrations of MPTOB291, tubacin, nexturastat A, and tubastatin A for 2 days. After treatment, the proportion of surviving cells was estimated using the MTT assay. Quantitative result (relative to DMSO) from three independent experiments is shown. B P1s cells (a), U87MG (b), U87MG-R (c), A172 (d), A172-R (e), P3 (f), and P3-R (g) were treated with the indicated concentrations of MPT0B291 for 4 days. After treatment, the proportion of surviving cells was estimated using the MTT assay. Data (relative to Day 0) are representative of three independent experiments and presented as the mean \pm SEM. C Tumors with DMSO or $10 \mathrm{mg} / \mathrm{kg}$ MPTOB291 treatment from SCID mice implanted with U87MG cells for 6 weeks are shown (a), and quantitative result of tumor weights is shown (b). D U87MG (a) or P1s cells (b) inoculated orthotopic mice were randomly grouped and treated with DMSO, $10 \mathrm{mg} / \mathrm{kg} \mathrm{TMZ} \mathrm{(for} \mathrm{P1s} \mathrm{cells} \mathrm{inoculated} \mathrm{orthotopic} \mathrm{mice)} \mathrm{or}$ $10 \mathrm{mg} / \mathrm{kg}$ MPTOB291 three days/week thereafter from day 5. Results of the Kaplan-Meier curve for the duration of survival in the control group (blue line) and MPTOB291-treated group (red line) are shown. E $\gamma \mathrm{H} 2 \mathrm{AX}$ levels in xenograft tumors were studied using IHC staining (a), and quantified (b) using ImageJ analysis with the IHC profiler score $(n=10)$. H\&E staining of xenograft tumors (c) for nuclei counting using ImageJ analysis (d).

in the promoter of DDR genes and was significantly upregulated in primary and recurrent GBMs (Supplementary Fig. S2). Using the same analysis approach, we observed that Sp1 was also one of the candidate TFs involved in the regulation of RAD51 (Fig. 5B, C).

Our recent study has indicated that MPTOB291-mediated Sp1 acetylation shows a decreased DNA binding ability of Sp1 resulting in altered expression of downstream genes [23]. In addition, we have found that the depletion of Sp1 significantly decreases HR efficiency [27]; however, the detailed mechanism is unknown. Analysis of Sp1-ChIP-seq data revealed that Sp1 is bound on the promoter region of RAD51 (Fig. 5D). Overexpression of Sp1 reversed the effect of MPTOB291-mediated RAD51 downregulation (Fig. 5E). Furthermore, knockdown of HDAC6 downregulated RAD51 expression in both P3 and P3-R cells; however, it did not decrease significantly in Sp1-overexpressed and -knockdown cells (Fig. 5F). The expression of RAD51 was also downregulated in mithramycin $A$ (Sp1 inhibitor)-treated cells and an accompanied increase was observed in $\mathrm{\gamma H} 2 \mathrm{AX}$ levels (Fig. 5G). These results indicate that $\mathrm{Sp} 1$ is involved in the regulation of DDR by controlling the expression level of RAD51.

The HDAC6/Sp1 axis may not target the RAD51 promoter alone. In addition to RAD51, 13 DDR gene promoter sequences were also occupied by Sp1 (Supplementary Fig. S3). We integrated multiomics data from the Sp1-binding profile, and gene expression profiles of clinical GBM samples (TCGA-GBM dataset) and MPTOB291-treated GBM cells. We identified that 8 DDR genes (including RAD51, CHEK1, GEN1, EXO1, TDG, NEIL3, RAD54L, and DDB2) shows a potential to be regulated by $\mathrm{HDAC} / \mathrm{Sp} 1$ (Supplementary Table S1). Supporting this finding, the expression of these DDR genes was significantly downregulated by MPTOB291 in both GBM and TMZ-resistant GBM cells (Fig. 6A and Supplementary Fig. S4). Moreover, the effects of MPTOB291mediated DDR gene inhibition (Fig. 6A) and DNA damage induction (Fig. 6B) were impaired in Sp1-overexpressed cells. The HDAC6 signaling leading to DDR gene expression is also confirmed by the knockout approach with HDAC6 to overcome the limitation of the chemical inhibition of HDAC6 using MPTOB291 (Supplementary Fig. S5). Further analysis of the clinical outcome data showed that higher expression levels of these DDR genes in high-grade and/or low-grade gliomas are significantly associated with decreased survival (Fig. 6C and Supplementary Fig. S6). Taken together, these results highlight the importance of the HDAC6/Sp1 axis in the DDR pathway, suggesting that targeting HDAC6/Sp1 signaling is an effective strategy against GBM and recurrent GBM with higher DNA repair capacity.

\section{DISCUSSION}

Induction of DNA damage has served as a therapeutic strategy for several cancers. Apart from chemotherapeutic drugs that exert cytotoxic effects, developing targeted therapy against DDR signaling is one of the current treatment strategies. There are two types of Food and Drug Administration-approved DDR inhibitors (PARP inhibitor and topoisomerase inhibitors), which are applied in cancer therapy [28]. Olaparib (Lynparza), a PARP inhibitor that has recently been approved for the treatment of metastatic breast cancer harboring BRCA1 or BRCA2 mutations, represents the first medicine based on the precision medicine concept. The median progression-free survival of patients with HER2-negative and BRCA mutation receiving Lynparza is 7 months compared to that of 4.2 months in patients taking chemotherapy alone [29]. Therefore, understanding the patients' genetic makeup or gene expression profile is crucial in clinical practice.

Recent advances in genomics, bioinformatics, and big biodata analysis have been the driving forces to implement precision medicine in clinical practice [30]. Biological databases such as Gene Expression Omnibus and TCGA integrate and provide enormous amounts of omics data, serving as crucially important resources for scientists [31]. To elucidate the gene expression profiles in patients with GBM, we obtained transcriptome NGS data from TCGA. The TCGA-GBM dataset was obtained from a total of 142 specimens: 5 normal tissues, 124 primary tumor tissues, and 13 recurrent tumor tissues. After performing systematic analysis on the 177 well-defined DDR genes, we identified several DDR genes that were dysregulated in clinical GBM cases. Even though some of the DDR genes, such as enhancer of zeste 2 polycomb repressive complex 2 subunit $(\mathrm{EZH} 2)$ and human telomerase reverse transcriptase (hTERT), have been recently reported to be altered in primary GBM [11], most studies focus on one of the DDR genes of their interest. Since cancer is a complex disease, changes in multiple gene expression contribute to tumor progression and the concept of multitarget drugs has also been applied in the clinical setting [32]. Understanding pathway-targeteddrug-disease relationships is required. To this end, we performed bioinformatics studies to identify the promoter region of GBMassociated DDR genes for detection of upstream regulators. We had found that Sp1 is most potential to regulate DDR gene expression, and this result explains the mechanism how Sp1 regulates DNA repair in GBM [27]. A detailed study of HDAC6/Sp1DDR gene regulation may provide new insights into GBM therapy because targeting this pathway may have an equal effect to that of multitarget drugs. Indeed, Sp1 depletion by Sp1 or HDAC6 inhibitors resulted in decreased expression of RAD51, CHEK1, EXO1, RAD54L, and GEN1, and then caused defective HR repair. Additionally, signaling of BER and NER is potentially influenced through the downregulation of NEIL3, TDG, and DDB2 expression (Fig. 6D). These gene expression statuses were also strongly correlated with the prognosis of the glioma patients (Fig. 6C); particularly, low expression levels were observed in patients with IDH1 mutation (Supplementary Fig. S7). Although IDH1 mutation has dual effects, acting either as an oncogene or as a tumor suppressor gene in glioma [7, 33], patients with IDH mutation exhibited better prognosis than those with wild-type IDH1 [34, 35] (Supplementary Fig. S8). Inhibition of the HDAC6/Sp1 signaling pathway could reverse DDR gene expression from the wild-type form to the mutated form of IDH1 and exhibited better prognosis in the clinical setting. Taken together, the HDAC6/Sp1 axis is a critical pathway toward advancing treatment for GBM. 
A
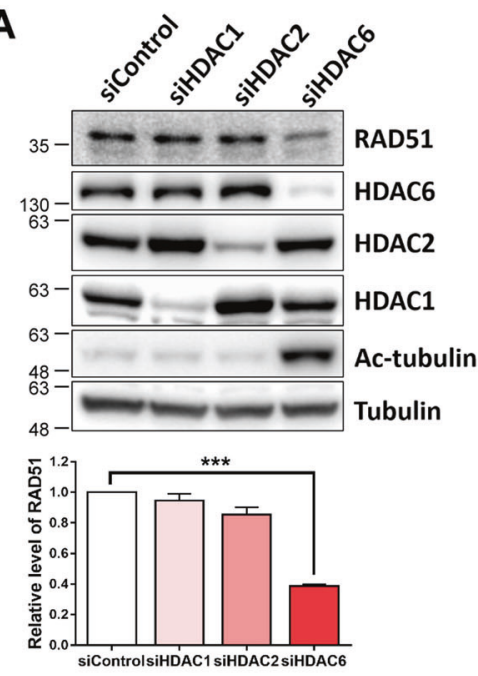

D

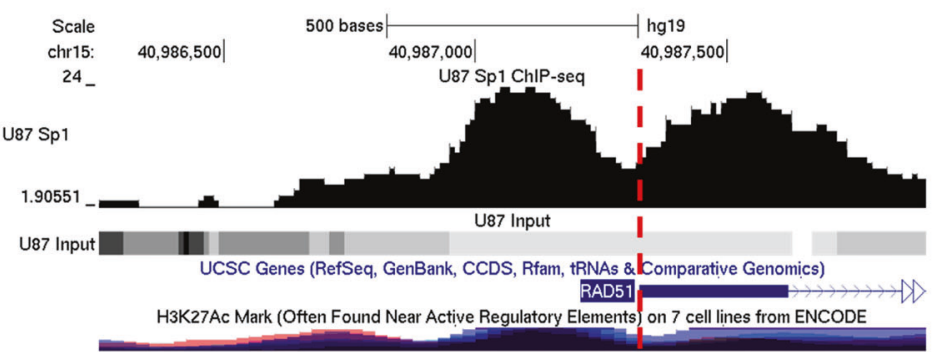

F

(a) GFP-Sp1 + + - - -

a) $\mathrm{siSp} 1 \quad-\quad-\quad++$

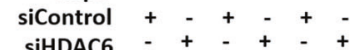

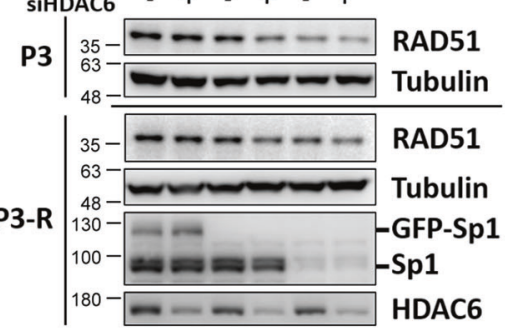

G

(a)

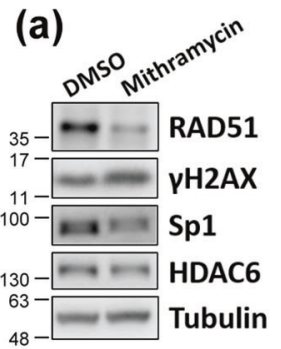

B

C

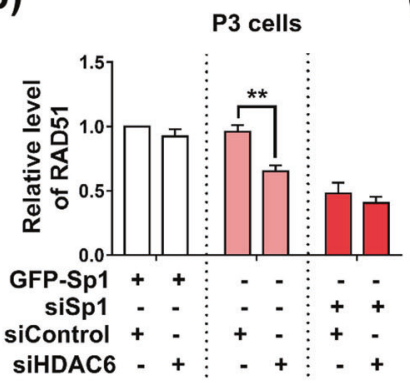

(c) (b)

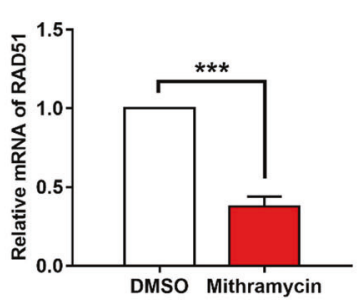

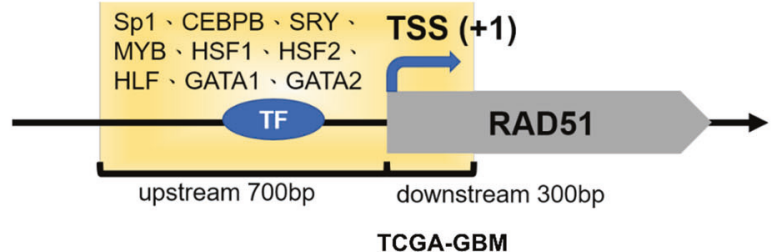
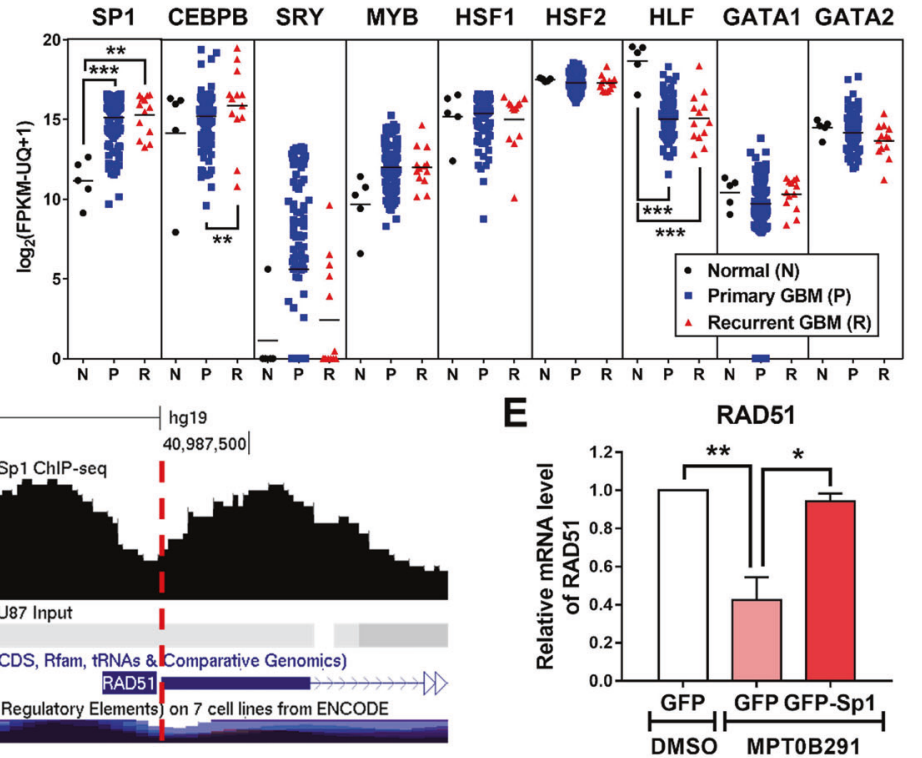

(c)

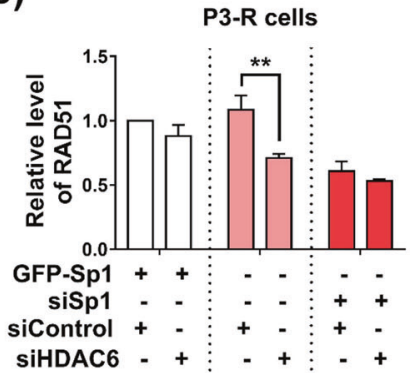

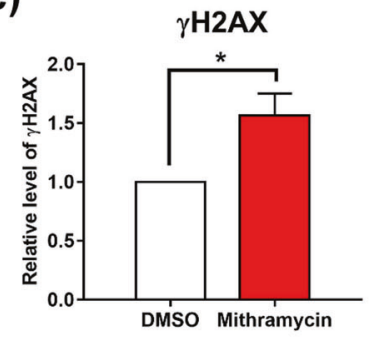

Fig. 5 HDAC6 regulates RAD51 expression through Sp1. A U87MG cells were transfected with control, HDAC1, HDAC2, and HDAC6 siRNA, respectively, for $72 \mathrm{~h}$. The protein levels of RAD51, HDAC1, HDAC2, HDAC6, Ac-tubulin, and tubulin were analyzed by western blotting (upper panels). Result of the quantitative analysis (normalized to tubulin) of RAD51 from three independent experiments is shown (lower panel). B Schematic diagram shows the promoter region used for transcription factor-binding analysis. TSS transcription start site. C The mRNA expression levels of SP1, CEBPB, SRY, MYB, HSF1, HSF2, HLF, GATA1, and GATA2 in normal brain, primary, and recurrent tumor samples (TCGAGBM dataset). D Distributions of Sp1-ChIP-seq reads mapped to the promoter region of RAD51. Visualization of the Sp1-ChIP-Seq data using the UCSC Genome Browser on Human (hg19) Assembly (https://genome.ucsc.edu/). The red dash line indicates TSS. E U87MG cells were treated with DMSO or $10 \mu \mathrm{M}$ MPTOB291 following transfection with GFP or GFP-Sp1 plasmid. On the next day of treatment, the mRNA expression of RAD51 in cells was analyzed using qPCR. Quantitative result (normalized to GAPDH) from three independent experiments is shown. F P3 and P3-R cells were transfected with GFP-Sp1, siSp1, siControl, and siHDAC6, respectively, for $72 \mathrm{~h}$. The protein levels of RAD51, Sp1, HDAC6, and tubulin were analyzed using western blotting (a). Quantitative results (normalized to tubulin) of RAD51 in P3 cells (b) and P3$\mathrm{R}$ cells (c) from three independent experiments are shown. G P3-R cells were treated with $10 \mu \mathrm{M}$ mithramycin A for $24 \mathrm{~h}$. The protein levels of RAD51, $\gamma \mathrm{H} 2 \mathrm{AX}$, Sp1, HDAC6, and tubulin were analyzed by western blotting (a). Quantitative results (normalized to tubulin) of RAD51 (b) and $\gamma \mathrm{H} 2 \mathrm{AX}$ (c) from three independent experiments are shown. 

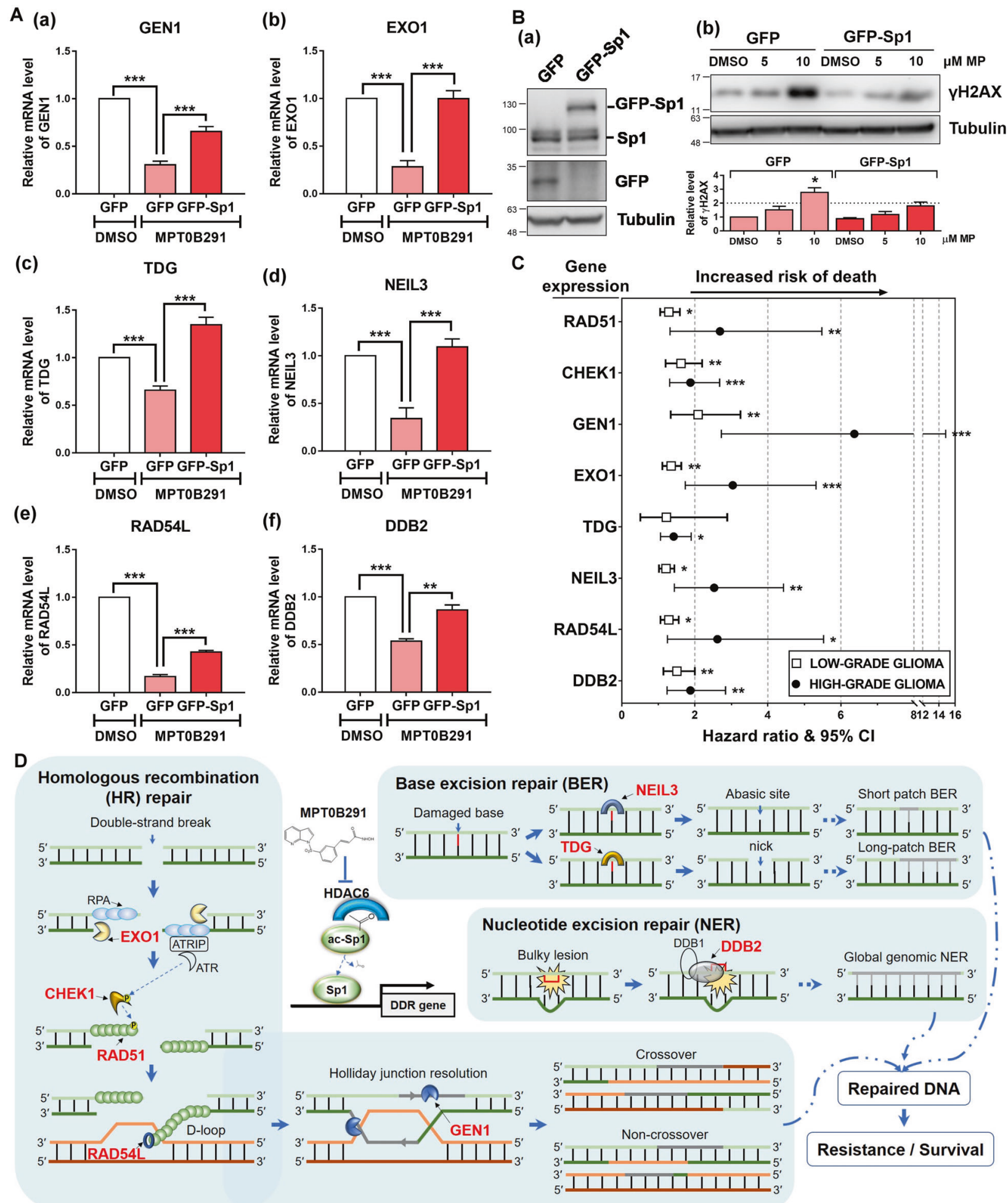

Fig. 6 Sp1 regulates DDR gene expression and impairs MPTOB291-induced DNA damage. A U87MG cells were treated with DMSO or $10 \mu \mathrm{M}$ MPTOB291 following transfection with GFP or GFP-Sp1 plasmid. One day after treatment, the mRNA expression of GEN1 (a), EXO1 (b), TDG (c), NEIL3 (d), RAD54L (e), and DDB2 (f) in cells was analyzed by qPCR. Quantitative results (normalized to GAPDH) from five independent experiments are shown. B A172 cells were transfected with GFP or GFP-Sp1, and treated with the indicated dosage of MPTOB291 for $24 \mathrm{~h}$. The protein levels of GFP-Sp1, Sp1, GFP (a), $\gamma \mathrm{H} 2 \mathrm{AX}$ and tubulin (b) were analyzed by western blotting. Quantitative result (normalized to tubulin) of $\gamma \mathrm{H} 2 \mathrm{AX}$ from three independent experiments is shown (lower panel). C Forest plots showing hazard ratios for the risk of death in patients with low-grade and high-grade glioma having higher expression of the indicated gene(s). The lines on both sides denote $95 \%$ confidence intervals. All the original data (Kaplan-Meier curve) were obtained from PROGgeneV2 database [51] (Supplementary Fig. S6). Hazard ratios above one indicate a poor outcome. D Schematic diagram shows that MPTOB291 induces DNA damage and cell death through the HDAC6/Sp1-mediated DDR gene regulation. 
Disruption of HDAC activities has been achieved with targeted therapy in different cancer types [36]. In GBM clinical trials, including SAHA combined with chemoradiotherapy for newly diagnosed GBM (NCT00731731), as well as SAHA plus bevacizumab (NCT01738646), panobinostat (PS) plus bevacizumab (NCT00859222), and romidepsin (NCT00085540) for recurrent GBM, had not improved the progression-free survival at 6 months or median overall survival. However, several HDAC inhibitor-associated clinical trials are still ongoing. In addition to the development of the isoform- or class-specific HDAC inhibitors, advances in brain-targeted delivery strategies could be considered important for the treatment of patients with GBM. Many challenges exist, such as cancer stemness, the blood-brain barrier (BBB) and drug-efflux pumps. More recently, we have provided evidence that a novel HDAC6 inhibitor, MPTOB291, inhibits the growth of stem-like/drug-resistant GBM cells and has better BBB permeability values than caffeine [23]. Thus, MPTOB291 may be a good treatment option for patients with GBM. A recent study showed that reversal of gene expression abnormalities correlates with drug efficacy in several diseases including breast, liver, and colon cancers [37]. Although Gobin et al. have identified DNA repair and cell cycle gene expression signatures for the classification of clinical samples using the nCounter technology [38], they have not provide any candidate compound that could be applied for clinical use. In the present study, we provided a list of abnormal DDR gene expression in GBM using the TCGA-GBM dataset big biodata analytics. Comparison of the expression of these genes between MPTOB291-treated samples and patients with GBM revealed a negative correlation (Pearson's $r=-0.412$ ) (Supplementary Fig. S1B), indicating that inhibition of HDAC6 by MPTOB291 has a potential to reverse the abnormalities of DDR genes in GBM. In more detail, RAD51, showed to be the most influenced DDR gene following MPTOB291 treatment (Fig. 2A), has been associated with radioresistance in GBM stem cells [39]. HDAC inhibitors, including SAHA and valproic acid (VPA), could reduce the expression levels of RAD51 [40]; however, the detailed mechanisms remain unclear. In the present study, we found that only HDAC6 inhibition can downregulate RAD51 expression through Sp1-mediated transcriptional regulation (Fig. 5). A study on acute myelogenous leukemia has indicated that HDAC inhibition by SAHA or PS results in the downregulation of RAD51 through miR-182 regulation [41]. It is also reported that Sp1 can also regulate miR-182 expression [42]. These observations raise the possibility that RAD 51 seems to be controlled by Sp1 at both transcriptional and post-transcriptional levels, highlighting the importance of $\mathrm{Sp} 1$ in RAD51-mediated HR repair.

In conclusion, our study uncovers the HDAC6/Sp1 signaling axis as an important pathway for the protection of GBM cells against DNA damage. HDAC6 inhibitors may serve as potential lead compounds for GBM therapy. These data could provide a foundation for clinical practice in the future.

\section{MATERIALS AND METHODS \\ Cell culture}

All cells, including U87MG (ATCC HTB-14, Manassas, VA), A172 (ATCC CRL1620), P3 (patient-derived primary GBM) [23], and their respective TMZresistant cells, as well as P1s (patient-derived chemo-resistant GBM) and primary mouse glial cells, were maintained in Dulbecco's modified Eagle's medium (DMEM; Gibco, Waltham, MA) supplemented with $10 \%$ fetal bovine serum (Gibco) at $37^{\circ} \mathrm{C}$ in a humidified atmosphere containing $5 \%$ $\mathrm{CO}_{2}$. Details of the isolation of patient-derived GBM cells are described previously $[23,27]$.

HDAC6 knockout (KO)-U87MG cells (commercialized KO cell line was prepared using clustered regularly interspaced short palindromic repeats strategy under the assistance of Biotools Co., Ltd. (New Taipei City, Taiwan)).
For preparing primary mouse glial cell cultures, cortices from postnatal day 0 (P0) to P1 mouse pups were dissociated by trituration and digestion with $10 \mathrm{U} / \mathrm{ml}$ trypsin (Gibco) for $30 \mathrm{~min}$ at $37^{\circ} \mathrm{C}$, and filtered through a $70 \mu \mathrm{m}$ nylon mesh cell strainer (Corning Incorporated, Corning, NY). Cells were plated onto cell culture plates coated with $50 \mu \mathrm{g} / \mathrm{ml}$ poly-L-lysine (Sigma-Aldrich, Temecula, CA). The mixed glial cultures were purified one week after isolation. Culture plates were taped onto a shaker inside an incubator and shaken at $150 \mathrm{rpm}$ for $2 \mathrm{~h}$. The supernatant was removed and cells were maintained in the DMEM culture medium. This procedure allowed cultures with a mixture of primary glial cells. Experiments are conducted under a protocol approved by the Joint Institutional Review Board of Taipei Medical University (Taipei, Taiwan) with the registration numbers (Nos. 201006011 and 201402018) and by the Institutional Animal Care and Use Committee (IACUC) of National Health Research Institutes (NHRI; Miaoli, Taiwan) with the registration number (NHRI-IACUC-106010).

\section{Dataset collection and processing}

The publicly available next-generation sequencing (NGS) databases of human GBM transcriptome were obtained from TCGA (https://portal.gdc. cancer.gov). The FPKM-UQ values of 177 well-defined DDR genes (these genes were obtained from the DDRprot database [43]) expressed in all of the 142 specimens, which consisted of five normal, 124 primary tumor, and 13 recurrent tumor tissues, were selected for the analysis. The fold change in gene expression and the level of significance of DDR genes between normal and GBM tissues were calculated. Details of ChIP-seq profiling of Sp1 in U87MG and expression profiling of 177 well-defined DDR genes in MPTOB291-treated U87MG-R or P3-R cells were acquired from our previous study [23].

\section{Bioinformatics analysis}

For cluster analysis of DDR gene expression profiling and upstream transcription factor-binding profiling, hierarchical clustering was performed using the Euclidean distance method and average linkage [44]. Pathway and process network analyses were performed using MetaCore software (Clarivate Analytics, Philadelphia, PA). For upstream transcription factor-binding analysis, the promoter sequences (1000 bp upstream to $200 \mathrm{bp}$ downstream) of the clustered DDR genes (defined in Results) were obtained from the GenBank database and analyzed using TFBIND tools [45]. The criterion of matrix similarity was $>0.9$.

\section{Western blotting}

Sample lysates were prepared and western blotted as described previously [23]. Antibodies used were as follows: RAD51 [D4B10] rabbit monoclonal antibody (mAb) (\#8875, Cell Signaling Technology, Danvers, MA); CHEK1 [C1C2-6] rabbit polyclonal antibody (polyAb) (GTX100070, GeneTex, Hsinchu, Taiwan); $\gamma$ H2AX [EP854(2)Y] rabbit mAb (ab81299, Abcam, Cambridge, UK); Ac-tubulin [D20G3] rabbit mAb (\#5335, Cell Signaling Technology); HDAC6 [D2E5] rabbit mAb (\#7558, Cell Signaling Technology); tubulin [1E4C11] mouse mAb (\#66031-1-lg, Proteintech, Rosemont, IL); HDAC1 rabbit polyAb (H3284, Sigma-Aldrich); HDAC2 [3F3] mouse mAb (\#05-814, Millipore, Temecula, CA); Sp1 rabbit polyAb (\#07-645, Millipore); GFP rabbit polyAb (\#632592, Clontech, Mountain View, CA) and horseradish peroxidase-conjugated secondary antibodies (Santa Cruz Biotechnology, Heidelberg, Germany). The protein bands were detected using the ChemiDoc Touch Imaging System (Bio-Rad Laboratories, Hercules, CA) and recorded using Image Lab software (Bio-Rad Laboratories). The band intensities were quantified using Scion image software (Scion, Frederick, MD).

\section{Micronucleus assay}

U87MG cells were seeded onto coverslips (with a thickness of $0.17 \mathrm{~mm}$ ) and incubated with dimethyl sulfoxide (DMSO; Sigma-Aldrich) or MPTOB291 for $24 \mathrm{~h}$. After fixation with $4 \%$ paraformaldehyde (Sigma-Aldrich) in PBS for $15 \mathrm{~min}$ and permeabilization with $1 \%$ Triton X-100 (Sigma-Aldrich) for $5 \mathrm{~min}$, cells were mounted in ProLong Gold Antifade Mountant with DAPI (Invitrogen, Waltham, MA), and then photographed using an immunofluorescence microscope (Leica DM6000 B; Wetzlar, Germany).

\section{HR and NHEJ DNA repair assays}

For the HR assay [46], cells stably expressing HR reporter plasmid (DR-GFP) were used to measure the HR frequency. The DR-GFP cells were 
co-transfected at a 4:1 ratio using the I-Scel endonuclease (cleavage creates a DSB) and DsRed-Monomer (as control for transfection efficiency) plasmids. For the NHEJ assay [47], the NHEJ reporter was generated from the GFP vector by Nhel (New England Biolabs, Ipswich, Massachusetts) enzyme digestion. U87MG cells were co-transfected at a 2:1 ratio using the NHEJ reporter and DsRed-Monomer plasmids. Cells were harvested 2 days after transfection and subjected to flow cytometry analysis by Guava EasyCyte System (Millipore). Only DsRed-positive cells were analyzed for HR and NHEJ efficiency to circumvent possible differences in transfection efficiencies. Data were analyzed to reveal the percentage of GFP-positive cells relative to that of DsRed-positive cells. Data were set to $1 \%$ of the background level of GFP-positive cells in every internal control.

\section{MTT cell viability assay}

Cells were seeded onto a 24-well petri-dish at a density of $1.5 \times 104$ cells/ well. One day after culture (Day 0), the cells were treated with MPTOB291 at varying doses as indicated. For 3-(4,5-dimethylthiazol-2-yl)-2,5-diphenyltetrazolium bromide (MTT; Bionovas, Ontario, Canada) staining, the cells were incubated with fresh medium containing the MTT reagent (final concentration $0.5 \mathrm{mg} / \mathrm{ml}$ ) at $37^{\circ} \mathrm{C}$ for $20 \mathrm{~min}$, and then, the MTT medium was exchanged using $300 \mu \mathrm{l}$ of DMSO to dissolve the MTT formazan crystals in cells. The absorbance of the supernatant of DMSO extract was measured at a wavelength of $550 \mathrm{~nm}$ with a reference wavelength of $655 \mathrm{~nm}$ using the iMark Microplate Absorbance Reader (Bio-Rad Laboratories).

\section{Experimental animals}

The animal experiments were approved by the IACUC (NHRI-IACUC106010) at the NHRI. Five- to six-week-old male immunodeficient NOD. CB17-Prkdc ${ }^{\text {scid } / J N a r l ~(S C I D) ~ m i c e ~ w e r e ~ p u r c h a s e d ~ f r o m ~ B i o L A S C O ~(T a i p e i, ~}$ Taiwan) and maintained at the animal facility of NHRI. For subcutaneous inoculation, U87MG $\left(10^{6}\right.$ cells) were suspended in $100 \mu$ of DMEM and implanted into the back of SCID mice. Tumor weight was determined from tumor tissue surgically excised from the back of SCID mice on day 42 following implantation. For the generation of an orthotopic model, a skull burr hole was created in the right frontal brain area. An ultrafine needle was then inserted to a depth of $3 \mathrm{~mm}$ using a stereotactic guiding device, and then, $5 \times 10^{5}$ U87MG or P1s cells (suspended in $3 \mu$ l of DMEM) were injected slowly to the mouse brain. In each experiment, all animals received surgically implanted GBM cells from the same researchers and on the same day. Total operating time of surgical procedures in 20 mice are controlled generally for $2 \mathrm{~h}$ and GBM cells were placed on the ice to avoid time effects on cell viability. Animals in the same cages were randomly and equally assigned to treatments to avoid cage effects and other possible biases linked to the timing of implantation and intervention. Administration of MPTOB291, TMZ, or vehicle (DMSO) via an intraperitoneal injection was initiated on day 5 after implantation (MPTOB291 was administered $8 \mathrm{~h}$ after TMZ injection in the P1s implanted group) and three times per week throughout the duration of the experiment. The scheduled treatment was interrupted when body weight loss was $>10 \%$ and re-initiated after weight recovery.

\section{Immunohistochemistry}

Xenograft tumors were fixed in $10 \%$ formaldehyde for $48 \mathrm{~h}$, dehydrated and embedded in paraffin. Sections were cut and stained with hematoxylin and eosin (H\&E). Moreover, limmunohistochemical (IHC) analysis was performed, the details of which are described in our previous study [48]. Briefly, blocked histological sections were stained using anti- $\mathrm{H} 2 \mathrm{AX}$ antibody. Immunoreactivity was detected using the DAB substrate kit (Vector Laboratories, Burlingame, CA). The expression level was quantified using the IHC profiler plugin in ImageJ software [49].

\section{Transient transfection}

Cells were used for transfection with plasmids (the manufacturing process has been described previously [50]) including pEGFP (GFP) and pEGFP-Sp1 (GFP-Sp1) using the GenJet reagent (SignaGen Laboratories, Rockville, MD) and commercial gene-specific SMARTpool short-interfering RNAs (Dharmacon, Lafayette, CO) for HDAC1 (siHDAC1), HDAC2 (siHDAC2), HDAC6 (siHDAC6), Sp1 (siSp1), and nontargeting siRNAs control (siControl) using Lipofectamine RNAiMAX (Invitrogen) according to the manufacturer's protocol. The transfection efficiency was confirmed by western blotting analysis.

\section{Quantitative PCR}

Total RNA was isolated using the TRIzol Reagent (Invitrogen) and Direct-zol RNA Miniprep Kits (Zymo Research, Irvine, CA) according to the manufacturer's protocol, and $1 \mu \mathrm{g}$ of RNA was reverse transcribed using the PrimeScript ${ }^{\text {TM }}$ RT Reagent Kit (TaKaRa, Mountain View, CA). Quantitative $\mathrm{PCR}$ (qPCR) was then performed using a mixture containing CDNA with SYBR Green Master Mix (Bio-Rad Laboratories) and gene-specific primers (Genomics, New Taipei City, Taiwan): RAD51, forward primer (F), 5'GGGGTGGAGGTGAAGGAAAG-3' and reverse primer (R), 5'-TGTTCTGTAA AGGGCGGTGG-3'; GEN1, F, 5'-AGCCCCACCTCAGGA ACTTA-3' and R, 5'-G CACACATGGCTTCAGCTTC-3'; EXO1， F， 5'-CGGGCCAACAATACCTTCCT-3' and R, 5'-TTGAATGGGCAGGCATAGCA-3'; TDG, F, 5'-TGCCAGAAGAAGTTCCAGCC-3' and R, 5'-ATCGGGGAGAGTCTTGGTCA-3'; NEIL3, F, 5'-GCCTCT GCATTCTCCGAGTT-3' and R, 5'-CCCATITCTGCCCACTGGA-3'; RAD54L, F, 5'-CGAGCATTGGGCCTGAAAAG-3' and R, 5'-TGAGGCCGCAAAACCTTACT-3'; DDB2, F, 5'-CCCTTTGACAGGAGGGCTAC-3' and R, 5'-AACTTCAGCCCAGTGATGCT-3'; GAPDH， $\mathrm{F}$, 5'-GAGTCAACGGATTTGGTCGT-3' and $\mathrm{R}, 5^{\prime}$ TTGATITTGGAGGGATCTCG-3'. mRNA expression levels of the indicated genes were measured using the StepOnePlus ${ }^{\text {TM }}$ Real-Time PCR System (Applied Biosystems, Waltham, MA). The relative mRNA level was calculated using the comparative cycle threshold $(\mathrm{Ct})$ method $(\Delta \Delta \mathrm{Ct})$ and normalized with the expression of GAPDH.

\section{Statistical analyses}

Statistical analyses of two groups of data from western blotting, micronucleus assay, HR/NHEJ DNA repair assays, MTT assay, tumor weight of subcutaneously inoculated mice, RNA-seq data of TCGA-GBM dataset, qPCR, etc. were carried out using Student's $t$-test with a two-tailed distribution. Multiple groups of data from the MTT assay and western blotting were analyzed by two-way analysis of variance with subsequent Tukey's multiple comparison test. The Gehan-Breslow-Wilcoxon test was used to compare the survival curves (Kaplan-Meier curve) of orthotopic GBM mice and patients with high-grade or low-grade gliomas. The Chisquare test was used to analyze IHC results. The DDR gene expression relationships between TCGA-GBM-dataset and MPTOB291-treated GBM cells were examined using the Pearson's correlation coefficient (PCC). An analysis of variance test is performed to determine whether genes (the RNA sequencing data related to glioma in Chinese Glioma Genome Atlas database) with significantly altered expression in the IDH1 mutant with respect to wild-type. Quantitative data (bar chart) are shown as mean \pm SEM. A value of $p<0.05$ was considered statistically significant $\left({ }^{*} p<0.05\right.$; ${ }^{* *} p<0.01 ;{ }^{* * *} p<0.001$ )

\section{DATA AVAILABILITY}

All data supporting the findings of this study are available within the article and supplementary data.

\section{REFERENCES}

1. Touat M, Idbaih A, Sanson M, Ligon KL. Glioblastoma targeted therapy: updated approaches from recent biological insights. Ann Oncol. 2017;28:1457-72.

2. Cohen MH, Johnson JR, Pazdur R. Food and Drug Administration Drug approval summary: temozolomide plus radiation therapy for the treatment of newly diagnosed glioblastoma multiforme. Clin Cancer Res. 2005;11:6767-71.

3. Lee SY. Temozolomide resistance in glioblastoma multiforme. Genes Dis. 2016;3:198-210.

4. Hegi ME, Diserens AC, Gorlia T, Hamou MF, de Tribolet N, Weller M, et al. MGMT gene silencing and benefit from temozolomide in glioblastoma. N. Engl J Med. 2005;352:997-1003.

5. Chang KY, Hsu TI, Hsu CC, Tsai SY, Liu JJ, Chou SW, et al. Specificity protein 1-modulated superoxide dismutase 2 enhances temozolomide resistance in glioblastoma, which is independent of $\mathrm{O}(6)$-methylguanine-DNA methyltransferase. Redox Biol. 2017;13:655-64.

6. Molenaar RJ, Maciejewski JP, Wilmink JW, van Noorden CJF. Wild-type and mutated IDH1/2 enzymes and therapy responses. Oncogene. 2018;37:1949-60.

7. Huang LE. Friend or foe-IDH1 mutations in glioma 10 years on. Carcinogenesis. 2019;40:1299-307.

8. Wang $\mathrm{P}, \mathrm{Wu} \mathrm{J}, \mathrm{Ma} \mathrm{S}$, Zhang L, Yao J, Hoadley KA, et al. Oncometabolite D-2hydroxyglutarate inhibits ALKBH DNA repair enzymes and sensitizes IDH mutant cells to alkylating agents. Cell Rep. 2015;13:2353-61.

9. Sulkowski PL, Corso CD, Robinson ND, Scanlon SE, Purshouse KR, Bai $H$, et al. 2-Hydroxyglutarate produced by neomorphic IDH mutations suppresses 
homologous recombination and induces PARP inhibitor sensitivity. Sci Transl Med. 2017;9:eaal2463.

10. Jackson SP, Bartek J. The DNA-damage response in human biology and disease. Nature. 2009;461:1071-8.

11. Erasimus $\mathrm{H}$, Gobin M, Niclou S, Van Dyck E. DNA repair mechanisms and their clinical impact in glioblastoma. Mutat Res Rev Mutat Res. 2016;769:19-35.

12. Annovazzi L, Mellai M, Schiffer D. Chemotherapeutic drugs: DNA damage and repair in glioblastoma. Cancers (Basel). 2017;9:57.

13. Sousa JF, Serafim RB, Freitas $L M$, Fontana CR, Valente V. DNA repair genes in astrocytoma tumorigenesis, progression and therapy resistance. Genet Mol Biol. 2019;43:e20190066.

14. Lesueur P, Lequesne J, Grellard JM, Dugue A, Coquan E, Brachet PE, et al. Phase I/ lla study of concomitant radiotherapy with olaparib and temozolomide in unresectable or partially resectable glioblastoma: OLA-TMZ-RTE-01 trial protocol. BMC Cancer. 2019;19:198.

15. Zhu J, Coyne CB, Sarkar SN. PKC alpha regulates Sendai virus-mediated interferon induction through HDAC6 and beta-catenin. EMBO J. 2011;30:4838-49.

16. Williams KA, Zhang $M$, Xiang S, Hu C, Wu JY, Zhang S, et al. Extracellular signalregulated kinase (ERK) phosphorylates histone deacetylase 6 (HDAC6) at serine 1035 to stimulate cell migration. J Biol Chem. 2013;288:33156-70.

17. Lafarga $V$, Mayor $F \mathrm{Jr}$, Penela $P$. The interplay between $G$ protein-coupled receptor kinase 2 (GRK2) and histone deacetylase 6 (HDAC6) at the crossroads of epithelial cell motility. Cell Adh Migr. 2012;6:495-501.

18. Watabe $M$, Nakaki T. Protein kinase CK2 regulates the formation and clearance of aggresomes in response to stress. J Cell Sci. 2011;124:1519-32.

19. Pugacheva EN, Jablonski SA, Hartman TR, Henske EP, Golemis EA. HEF1dependent Aurora $A$ activation induces disassembly of the primary cilium. Cell. 2007;129:1351-63.

20. Milazzo G, Mercatelli D, Di Muzio G, Triboli L, De Rosa P, Perini G, et al. Histone deacetylases (HDACs): evolution, specificity, role in transcriptional complexes, and pharmacological actionability. Genes (Basel). 2020;11:556.

21. Hubbert C, Guardiola A, Shao R, Kawaguchi $Y$, Ito A, Nixon A, et al. HDAC6 is a microtubule-associated deacetylase. Nature. 2002;417:455-8.

22. Wang Z, Hu P, Tang F, Lian H, Chen X, Zhang $Y$, et al. HDAC6 promotes cell proliferation and confers resistance to temozolomide in glioblastoma. Cancer Lett. 2016;379:134-42.

23. Yang WB, Hsu CC, Hsu TI, Liou JP, Chang KY, Chen PY, et al. Increased activation of HDAC1/2/6 and Sp1 underlies therapeutic resistance and tumor growth in glioblastoma. Neuro Oncol. 2020;22:1439-51.

24. Tsai YT, Wu CC, Ko CY, Hsu TI, Chang WC, Lo WL, et al. Correlation between the expression of cancer stem cell marker BMI1 and glioma prognosis. Biochem Biophys Res Commun. 2021;550:113-9.

25. Zhang M, Xiang SY, Joo HY, Wang L, Williams KA, Liu W, et al. HDAC6 deacetylates and ubiquitinates MSH2 to maintain proper levels of MutS alpha. Mol Cell. 2014;55:31-46.

26. Kim GW, Lee DH, Yeon SK, Jeon YH, Yoo J, Lee SW, et al. Temozolomide-resistant glioblastoma depends on HDAC6 activity through regulation of DNA mismatch repair. Anticancer Res. 2019;39:6731-41.

27. Yang WB, Chuang JY, Ko CY, Chang WC, Hsu TI. Dehydroepiandrosterone induces temozolomide resistance through modulating phosphorylation and acetylation of Sp1 in glioblastoma. Mol Neurobiol. 2019;56:2301-13.

28. O'Connor MJ. Targeting the DNA damage response in cancer. Mol Cell. 2015;60:547-60.

29. Kaufman B, Shapira-Frommer R, Schmutzler RK, Audeh MW, Friedlander M, Balmana J, et al. Olaparib monotherapy in patients with advanced cancer and a germline BRCA1/2 mutation. J Clin Oncol. 2015;33:244-50.

30. Raja R, Lee YS, Streicher K, Conway J, Wu S, Sridhar S, et al. Integrating genomics into drug discovery and development: challenges and aspirations. Pharm Med. 2017;31:217-33.

31. Zou D, Ma L, Yu J, Zhang Z. Biological databases for human research. Genomics Proteomics Bioinformatics. 2015;13:55-63.

32. Ramsay RR, Popovic-Nikolic MR, Nikolic K, Uliassi E, Bolognesi ML. A perspective on multi-target drug discovery and design for complex diseases. Clin Transl Med. 2018;7:3.

33. Dang L, Yen K, Attar EC. IDH mutations in cancer and progress toward development of targeted therapeutics. Ann Oncol. 2016;27:599-608.

34. Hartmann C, Hentschel B, Wick W, Capper D, Felsberg J, Simon M, et al. Patients with IDH1 wild type anaplastic astrocytomas exhibit worse prognosis than IDH1mutated glioblastomas, and IDH1 mutation status accounts for the unfavorable prognostic effect of higher age: implications for classification of gliomas. Acta Neuropathol. 2010;120:707-18.

35. Tateishi K, Wakimoto H, Cahill DP. IDH1 mutation and World Health Organization 2016 diagnostic criteria for adult diffuse gliomas: advances in surgical strategy. Neurosurgery. 2017;64:134-8.
36. Mottamal M, Zheng SL, Huang TL, Wang GD. Histone deacetylase inhibitors in clinical studies as templates for new anticancer agents. Molecules. 2015;20:3898-941.

37. Chen B, Ma L, Paik H, Sirota M, Wei W, Chua MS, et al. Reversal of cancer gene expression correlates with drug efficacy and reveals therapeutic targets. Nat Commun. 2017;8:16022.

38. Gobin M, Nazarov PV, Warta R, Timmer M, Reifenberger G, Felsberg J, et al. A DNA repair and cell-cycle gene expression signature in primary and recurrent glioblastoma: prognostic value and clinical implications. Cancer Res. 2019;79:1226-38.

39. McKenzie LD, LeClair JW, Miller KN, Strong AD, Chan HL, Oates EL, et al. CHD4 regulates the DNA damage response and RAD51 expression in glioblastoma. Sci Rep. 2019;9:4444.

40. Cornago M, Garcia-Alberich C, Blasco-Angulo N, Vall-llaura N, Nager M, Herreros J, et al. Histone deacetylase inhibitors promote glioma cell death by $\mathrm{G} 2$ checkpoint abrogation leading to mitotic catastrophe. Cell Death Dis. 2014;5:e1435.

41. Lai TH, Ewald B, Zecevic A, Liu C, Sulda M, Papaioannou D, et al. HDAC inhibition induces microRNA-182, which targets RAD51 and impairs HR repair to sensitize cells to sapacitabine in acute myelogenous leukemia. Clin Cancer Res. 2016;22:3537-49.

42. Yang WB, Chen PH, Hsu TI, Fu TF, Su WC, Liaw H, et al. Sp1-mediated microRNA182 expression regulates lung cancer progression. Oncotarget. 2014;5:740-53.

43. Andres-Leon E, Cases I, Arcas A, Rojas AM. DDRprot: a database of DNA damage response-related proteins. Database (Oxf). 2016;2016:baw123.

44. Jaskowiak PA, Campello RJ, Costa IG. On the selection of appropriate distances for gene expression data clustering. BMC Bioinformatics. 2014;15:S2

45. Tsunoda T, Takagi T. Estimating transcription factor bindability on DNA. Bioinformatics. 1999;15:622-30.

46. Sartori AA, Lukas C, Coates J, Mistrik M, Fu S, Bartek J, et al. Human CtIP promotes DNA end resection. Nature. 2007;450:509-14.

47. Seluanov A, Mao Z, Gorbunova V. Analysis of DNA double-strand break (DSB) repair in mammalian cells. J Vis Exp. 2010;43:2002.

48. Yang WB, Chen PH, Hsu TS, Fu TF, Su WC, Liaw $H$, et al. Sp1-mediated microRNA-182 expression regulates lung cancer progression. Oncotarget. 2014;5: 740-53.

49. Varghese $F$, Bukhari $A B$, Malhotra R, De A. IHC Profiler: an open source plugin for the quantitative evaluation and automated scoring of immunohistochemistry images of human tissue samples. PLoS ONE. 2014;9:e96801.

50. Chuang JY, Wang YT, Yeh SH, Liu YW, Chang WC, Hung JJ. Phosphorylation by c-Jun $\mathrm{NH} 2$-terminal kinase 1 regulates the stability of transcription factor Sp1 during mitosis. Mol Biol Cell. 2008;19:1139-51.

51. Goswami CP, Nakshatri H. PROGgeneV2: enhancements on the existing database. BMC Cancer. 2014;14:970.

\section{ACKNOWLEDGEMENTS}

We thank Prof. Hungjiun Liaw (Department of Life Sciences, National Cheng Kung University, Taiwan) for providing the HR reporter plasmids.

\section{AUTHOR CONTRIBUTIONS}

Investigation and validation: WBY, ACW, TIH, and JPL. Clinical specimen collection: WLL, KYC, PYC, and STY. Data curation: WBY, ACW, CYK, and JYC. Supervision: UK, TJK, RMC, WCC, CYK, and JYC. Funding acquisition: WCC and JYC. Writing draft: WBY, ACW, CYK, and JYC. All authors revised and approved the final version of the manuscript.

\section{FUNDING}

This work was supported by the Ministry of Science and Technology in Taiwan (grant numbers 110-2636-B-038-004, 110-2314-B-038-094-MY2, 110-2320-B-038-078, and 109-2320-B-182A-005-MY3), as well as by the Taipei Medical University (TMU110-AE1B02) and the TMU Research Center of Cancer Translational Medicine from The Featured Areas Research Center Program within the framework of the Higher Education funded by the Ministry of Education in Taiwan.

\section{ETHICS STATEMENT}

Experiments are conducted under a protocol approved by the Joint Institutional Review Board of Taipei Medical University (Taiwan) with the registration numbers (Nos. 201006011 and 201402018) and by the Institutional Animal Care and Use Committee (IACUC) of National Health Research Institutes (NHRI; Taiwan) with the registration number (NHRI-IACUC-106010). 
COMPETING INTERESTS

The authors declare no competing interests.

\section{ADDITIONAL INFORMATION}

Supplementary information The online version contains supplementary material available at https://doi.org/10.1038/s41419-021-04182-w.

Correspondence and requests for materials should be addressed to Chiung-Yuan Ko or Jian-Ying Chuang.

Reprints and permission information is available at http://www.nature.com/ reprints

Publisher's note Springer Nature remains neutral with regard to jurisdictional claims in published maps and institutional affiliations.
Open Access This article is licensed under a Creative Commons Attribution 4.0 International License, which permits use, sharing, adaptation, distribution and reproduction in any medium or format, as long as you give appropriate credit to the original author(s) and the source, provide a link to the Creative Commons license, and indicate if changes were made. The images or other third party material in this article are included in the article's Creative Commons license, unless indicated otherwise in a credit line to the material. If material is not included in the article's Creative Commons license and your intended use is not permitted by statutory regulation or exceeds the permitted use, you will need to obtain permission directly from the copyright holder. To view a copy of this license, visit http://creativecommons. org/licenses/by/4.0/.

(c) The Author(s) 2021 